\title{
Effects of Dietary Bitter Melon Extract on Growth Performance, Antioxidant Capacity, Inflammatory Cytokines Expression, and Intestinal Microbiota in Common Carp (Cyprinus carpio L.)
}

\author{
Chaobin Qin $\mathbb{C}^{1},{ }^{1}$ Junli Wang, ${ }^{2}$ Wenli Zhao, ${ }^{1}$ Daming Pi, ${ }^{1}$ Xiao Yan, ${ }^{1}$ and Guoxing Nie $\mathbb{C}^{1}$ \\ ${ }^{1}$ College of Fisheries, Henan Normal University, No. 46 Jianshe Road, Xinxiang 453007, China \\ ${ }^{2}$ College of Life Sciences, Henan Normal University, No. 46 Jianshe Road, Xinxiang 453007, China
}

Correspondence should be addressed to Guoxing Nie; niegx@htu.cn

Received 13 November 2021; Accepted 23 December 2021; Published 31 January 2022

Academic Editor: Erchao Li

Copyright ( 2022 Chaobin Qin et al. This is an open access article distributed under the Creative Commons Attribution License, which permits unrestricted use, distribution, and reproduction in any medium, provided the original work is properly cited.

\begin{abstract}
Bitter melon extract (BME), which contains a variety of natural bioactive substances, has diversified biological functions. The present study is aimed at evaluating the effects of dietary supplementation of BME on growth performance, antioxidant capacity, inflammatory cytokine expression, and intestinal microbiota in common carp (Cyprinus carpio L.). An 8-week feeding trial was conducted using four isonitrogenous and isoenergetic diets containing different levels of $\mathrm{BME}(0 \%, 0.25 \%, 0.5 \%$, and $1 \%$ for control, LBME, MBME, and HBME groups, respectively). The results showed that there were no significant differences in the growth performance of common carp among all groups, including final body weight (FBW), weight gain rate (WGR), specific growth rate (SGR), feed conversion ratio (FCR), and viscera index (VSI) $(p>0.05)$, but the condition factor (CF) was decreased by all BME treatments $(p<0.05)$. Serum superoxide dismutase (SOD) activity was significantly improved in all BME groups $(p<0.05)$, and serum catalase (CAT) activity was significantly higher in the MBME group $(p<0.05)$. Serum malondialdehyde (MDA) level was lowest while serum total antioxidant capacity (T-AOC) was highest in the HBME group $(p<0.05)$. Dietary BME supplementation inhibited proinflammatory cytokine (il-1 $\beta$ and il-8) mRNA expression, while antiinflammatory cytokine (il-6 and il-10) mRNA expressions were promoted $(p<0.05)$. Furthermore, principal coordinate analysis (PCoA) showed that there were no differences in bacterial communities among different groups, and Shannon index and Simpson index of intestinal microbiota were unaffected $(p>0.05)$. Whereas dietary BME reduced Firmicutes abundance and the ratio of Firmicutes to Bacteroidetes at phylum level, the relative abundance of streptococcaceae at family level, Streptococcus and Exiguobacterium at genus level were also decreased $(p<0.05)$. Overall, these results suggested that dietary supplementation of BME improved serum antioxidant capacity, anti-inflammatory activity, and modified intestinal microbiota in common carp.
\end{abstract}

\section{Introduction}

In recent years, global aquaculture industry has developed rapidly, but the diseases caused by various pathogens, including viruses, bacteria, fungi and parasites, lead to increasing mortality of aquatic farming animals and huge economical loss. The prevention and control of diseases are still one of the important challenges faced by intensive aquaculture. To overcome this problem, antibiotics, disinfectants, and therapeutic agents are commonly used to prevent diseases [1]. However, long-term or excessive use of these drugs has been widely criticized, and they could not only damage immune defense, increase drug residues, but also lead to increased drug resistance to pathogens in aquaculture [2]. Therefore, considerable interest has arisen in finding alternative natural substances that enhance the immunity of aquatic animals [3].

Application of plant extracts and herbal medicines in aquatic animals has received increasing attention worldwide, because they could be easily prepared, and has few side effects on aquatic animals as well as environment [4]. Plant extracts contain many types of active components, like glycosides, 
flavonoids, terpenoids, and alkaloids. Studies have identified their beneficial effects for aquatic animals, such as stimulating appetite, promoting growth, boosting immunity, and acting as antibacterial, antiviral, antifungal, and antiparasitic agents [5-7]. At present, many extracts derived from various plants as feed additives have been widely studied in aquatic animals. It was found that they improved antioxidant and immune responses in cultured fishes, such as extracts from mistletoe (Viscum album) [8], olive (Elaeagnus angustifolia) leaf [9], ginger (Zingiber officinale) [10], and hawthorn (Crataegus monogyna) fruit [11]. In addition, our previous studies showed that dietary supplementation of Rehmannia glutinosa root extract improved growth performance and immune response [12] of common carp, and dietary Chinese yam (Dioscorea opposita Thunb.) peel enhanced immunity of common carp by improving intestine defense barrier and modulating intestinal microflora [13].

Bitter melon (Momordica charantia), also known as bitter gourd or balsam pear, is a widely cultivated food vegetable in many areas around the world, including Asia, South America, Central America, and East Africa [14]. Although the fruit has a special bitter taste, it is very popular among different groups of people all over the world, which can be explained not only by its special taste but also by various biological activities that ordinary vegetables cannot provide [15]. Bitter melon is rich in bioactive compounds, including saponins, flavonoids, phenolic acids, cucurbitane triterpenoids, and triterpene glycosides [16]. Moreover, studies in mammals demonstrated that this plant has various biological activities, such as antioxidant, anti-inflammatory, antibacterial, and immunoregulatory activities, as well as antidiabetic, antiobesity, and anticancer activities [16]. In rats, oral administration of BME remarkably increased key antioxidant enzymes activities, including superoxide dismutase (SOD) and serum catalase (CAT) [17]. Dietary supplementation of bitter melon dry powder reduced proinflammatory cytokine levels, inhibited recruitment of inflammatory macrophages into adipose tissues, and ameliorated insulin resistance of mice fed with high-fat diet [18]. However, to the best of our knowledge, the properties of $\mathrm{BME}$ as a potential bioactive substance in aquaculture have not been studied.

Intestine is the largest and most complex ecosystem of living organisms. Plenty of microorganisms are parasitic or symbiotic with the intestine of healthy animals. The microorganisms and their metabolites are involved in many physiological activities of the host organism. Studies about intestinal microbiota in fish have increased dramatically over the past 10 years, and the composition and diversity of intestinal microbial community could be strongly influenced by diet changes [19]. It is believed that plant products can affect intestinal microbiota in different ways, and their interplays contribute to the bioavailability of active ingredients [20]. Previous studies have found that BME is involved in the regulation of intestinal microbiota in obese rats induced by dietary high-fat diet [21]. However, the effect of BME on fish intestinal microflora remains to be evaluated.

Common carp (Cyprinus carpio L.) is an important economical freshwater fish, which is widely farmed in Asia, especially in northern China. The present study is aimed at investigating the effects of dietary BME on growth performance, antioxidant capacity, inflammatory cytokine expression, and intestinal microbiota of common carp. For this purpose, BME was supplemented to the basal diet of common carp with different proportions, and an 8-week feeding trial was performed.

\section{Materials and Methods}

2.1. Diet Preparation. BME was purchased from Xi'an Xuhuang Bio-Tech Co., Ltd (Xi'an, China). The basal diet without BME was used as control diet, and the diets with different concentrations of $\mathrm{BME}(0.25 \%$ for $\mathrm{LBME}, 0.5 \%$ for MBME, and $1 \%$ for $\mathrm{HBME}$ ) were used as experimental diets, and the composition is given in Table 1. All ingredients were grounded into powder, sieved through an 80-mesh filter, blended in a mixer thoroughly, and then mixed with fish oil and soybean oil [22]. Finally, a proper amount of water was added to ensure the uniformity of granulation. The $2 \mathrm{~mm}$ diameter granulated feed was wet-extruded using a pelletizer and dried at air temperature. Then, all diets were sealed in plastic bags and stored at $-20^{\circ} \mathrm{C}$ until used.

2.2. Feeding Trial. Juvenile common carp was obtained from Aquaculture Base of Henan Normal University. The fish were temporary reared for 2 weeks in the outdoor cement tank $(400 \mathrm{~cm} \times 200 \mathrm{~cm} \times 120 \mathrm{~cm})$ provided with running freshwater. A total of 420 fish were randomly assigned into twelve $150 \mathrm{~L}$ indoor tanks (35 fish per tank) in a freshwater recirculation system with a water flow rate of approximately $2.5 \mathrm{~L} / \mathrm{min} /$ tank and divided into 4 groups (control, LBME, MBME, and HBME) with triplicate. After 2 weeks of acclimation with basal diet, the experimental fish in different groups was fed with prepared diets, respectively. Fish were fed 3 times per day $(8: 30,13: 00$, and 17:30) for 8 weeks according to $3 \%$ feeding rate, which was adjusted every two weeks. The water temperature was maintained as $24 \pm$ $1^{\circ} \mathrm{C}$, and dissolved oxygen was above $6.0 \mathrm{mg} / \mathrm{L}, \mathrm{pH} 7.5 \pm$ 0.5 , and total ammonia below $0.1 \mathrm{mg} / \mathrm{L}$.

2.3. Sampling Collection. After feeding experiment, fish were fasted for $24 \mathrm{~h}$ and anaesthetized with MS-222 $(100 \mathrm{mg} / \mathrm{L}$, Sigma, USA) before sampling. The whole fish population was sampled for final body weight (FBW), weight gain rate (WGR), specific growth rate (SGR), feed conversion ratio (FCR), and condition factor (CF), and 3 fish per tank were randomly sampled for viscera index (VSI). Blood were collected ( 3 fish per tank) with a $1 \mathrm{~mL}$ syringe via puncture of caudal vein and then centrifuged at $3000 \mathrm{rpm}$ for $10 \mathrm{~min}$ at $4^{\circ} \mathrm{C}$ to prepare serum samples, which were subsequently stored at $-80^{\circ} \mathrm{C}$ until analysis. As for gene expression analysis, samples of head kidney, spleen, and intestine were collected from 3 fish per tank, frozen in liquid nitrogen immediately, and then transferred to a $-80^{\circ} \mathrm{C}$ refrigerator until RNA extraction. The intestinal contents were carefully removed from two fish per tank by gently squeezing with sterile forceps in a clean bench. The samples were frozen 
TABLE 1: Formulation and proximate composition of experimental diets.

\begin{tabular}{|c|c|c|c|c|}
\hline \multirow{2}{*}{ Ingredients (\%) } & \multicolumn{4}{|c|}{ Diets $^{\mathrm{a}}$} \\
\hline & Control & LBME & MBME & HBME \\
\hline Soybean meal & 13 & 13 & 13 & 13 \\
\hline Fish meal & 17 & 17 & 17 & 17 \\
\hline Rapeseed meal & 13 & 13 & 13 & 13 \\
\hline Cotton meal & 12 & 12 & 12 & 12 \\
\hline Wheat grain & 26 & 26 & 26 & 26 \\
\hline Meat and bone meal & 5 & 5 & 5 & 5 \\
\hline Soybean oil & 5.5 & 5.5 & 5.5 & 5.5 \\
\hline Full-fat soybean & 1.5 & 1.5 & 1.5 & 1.5 \\
\hline $\mathrm{Ca}\left(\mathrm{H}_{2} \mathrm{PO}_{4}\right)_{2}$ & 2 & 2 & 2 & 2 \\
\hline Multivitamin ${ }^{\mathrm{b}}$ & 0.3 & 0.3 & 0.3 & 0.3 \\
\hline Multimineral $^{\mathrm{c}}$ & 2.5 & 2.5 & 2.5 & 2.5 \\
\hline Multibentonite & 1 & 1 & 1 & 1 \\
\hline Cellulose & 1.2 & 0.95 & 0.7 & 0.2 \\
\hline Bitter melon extract & 0 & 0.25 & 0.5 & 1 \\
\hline Total & 100 & 100 & 100 & 100 \\
\hline \multicolumn{5}{|c|}{ Proximate composition (\% dry weight basis) } \\
\hline Crude protein & 34.088 & 34.088 & 34.088 & 34.088 \\
\hline Crude lipid & 8.2025 & 8.2025 & 8.2025 & 8.2025 \\
\hline Crude fiber & 4.2935 & 4.2935 & 4.2935 & 4.2935 \\
\hline Nitrogen free extract & 2.9351 & 2.9351 & 2.9351 & 2.9351 \\
\hline Crude ash & 7.4915 & 7.4915 & 7.4915 & 7.4915 \\
\hline
\end{tabular}

${ }^{\mathrm{a}}$ Basal diet without BME was used as control diet, and the diets with different concentrations of BME (0.25\% for LBME, $0.5 \%$ for MBME, and $1 \%$ for HBME) were used as experimental diets. ${ }^{\mathrm{b}}$ Multivitamin (mg or IU/ $\mathrm{kg}$ diet): vitamin A, $1000 \mathrm{IU}$; vitamin $\mathrm{B} 1,5 \mathrm{mg}$; vitamin $\mathrm{B} 2,10 \mathrm{mg}$; vitamin $\mathrm{B} 5,20 \mathrm{mg}$; vitamin $\mathrm{B} 6,5 \mathrm{mg}$; vitamin B12, $0.5 \mathrm{mg}$; vitamin $\mathrm{C}$, $50 \mathrm{mg}$; vitamin D3, $2500 \mathrm{IU}$; vitamin E, $45 \mathrm{mg}$; pantothenate, $150 \mathrm{mg}$; folacin, $20 \mathrm{mg}$; biotin, $2.5 \mathrm{mg}$; inositol, $100 \mathrm{mg}$. ' Multimineral $(\mathrm{mg} / \mathrm{kg}$ diet): $\mathrm{Mg}: 350 \mathrm{mg}$; Fe: $150 \mathrm{mg}$; Zn: $80 \mathrm{mg}$; Mn, $25 \mathrm{mg}$; Cu: $5 \mathrm{mg}$; I, $0.5 \mathrm{mg}$; Co: $1.0 \mathrm{mg}$; Se, $0.2 \mathrm{mg}$.

in liquid nitrogen for quick freezing and stored at $-80^{\circ} \mathrm{C}$ until DNA extraction.

2.4. Determination of Antioxidant Indexes. The activities of serum antioxidant enzymes, including superoxide dismutase (SOD) and catalase (CAT), serum malondialdehyde (MDA) content, and total antioxidant capacity (T-AOC), were measured using commercial assay kits (Solarbio, China) according to the manufacturer's protocol, and the product codes are $\mathrm{BC} 0170, \mathrm{BC} 0205, \mathrm{BC} 0025$, and BC1315, respectively.

2.5. Gene Expression Analysis. Total RNA was extracted using RNAiso Plus (TaKaRa, 9109) according to the manufacturer's instruction. RNA purity and concentration were evaluated with a NanoDrop 2000C spectrophotometer (Thermo Scientific, USA), and RNA integrity was assessed by agarose gel electrophoresis. Then, $1 \mu \mathrm{g}$ of total RNA was used to synthesize cDNA using PrimeScript RT reagent Kit with gDNA Eraser (TaKaRa, RR047A) according to the manufacturer's protocol. RT-qPCR was performed using SYBR Green qPCR Mix (Dongsheng Biotech, P2092) with
LightCycler 480 II (Roche), and the procedures were described as our previous study [23]. Briefly, $10 \mu \mathrm{L}$ reactions contained $5 \mu \mathrm{L}$ SYBR Green qPCR Mix, $0.3 \mu \mathrm{L}$ of each primer $(10 \mu \mathrm{M}), 1 \mu \mathrm{L}$ of 10 -fold diluted $\mathrm{cDNA}$, and $3.4 \mu \mathrm{L}$ of water, and PCR amplification was initiated by denaturation at $94^{\circ} \mathrm{C}$ for $3 \mathrm{~min}, 40$ cycles of $95^{\circ} \mathrm{C}$ for $15 \mathrm{~s}, 56^{\circ} \mathrm{C}$ for $15 \mathrm{~s}$, and $72^{\circ} \mathrm{C}$ for $30 \mathrm{~s}$. The primer sequences for target genes and reference gene (18S RNA) are shown in Table 2. After verification of PCR efficiency of tested genes to be around $100 \%$ (Table 2 ), the relative gene expression was normalized to $18 \mathrm{~S}$ with $2^{-\Delta \Delta \mathrm{CT}}$ method.

2.6. Genomic DNA Extraction and Illumina HighThroughput Sequencing. The genomic DNA was extracted from the intestinal content samples using a genomic DNA extraction kit (QIAamp, Germany). Their concentrations and integrity were determined by NanoDrop 2000 and agarose-electrophoresis, respectively. Using the qualified DNA was used as template, and 338F $\left(5^{\prime}\right.$-ACTCCTACGGGAGG CAGCA- $\left.3^{\prime}\right)$ and 806R (5'-ATGCAGGGACTAC HVGGGTWTCTAAT- ${ }^{\prime}$ ) were used as primers, the V3V4 region of bacteria $16 \mathrm{~S}$ ribosomal RNA gene was amplified, and the amplicons were purified with a gel extraction kit (CW2302, CoWin Biosciences, China), and then sequenced by Majorbio Bio-Pharm Technology Co., Ltd., (Shanghai, China). The resulted sequences were clustered to operational taxonomic units (OTUs) with 3\% divergence (97\% similarity) using Parse (v.7.1, http://drive5.com/ uparse/) for further bioinformatics analysis. Chimeric sequences were identified and removed by UCHIME. The phylogenetic affiliation of each $16 \mathrm{~S}$ rRNA gene sequence was analyzed by RDP Classifier algorithm (version 2.2 http://sourceforge.net/projects/rdp-classifier/) against the Silva (SSU123) 16S rRNA database according to a confidence threshold of $70 \%$. According to species annotations, the number of sequences annotated to each classification was counted for each sample. The diversity indexes were analyzed using MOTHUR program (version 1.33.3, http:// www.mothur.org).

2.7. Statistical Analysis. The results were presented as mean \pm SEM. The variables were first submitted to normality tests and homoscedasticity tests using Shapiro-Wilk and Levene's test with SPSS 20.0 software. Then, statistical differences between groups were identified by one-way ANOVA followed by Duncan's multiple range tests. Probability of less than $0.05 \quad(p<0.05)$ was considered as statistically significant.

\section{Results}

3.1. Growth Performance. Growth performance parameters are shown in Table 3. Compared with basal diet (control group), dietary supplementation of BME with different proportions (LBME, MBME, and HBME groups) had no significant effect on growth performance of common carp, including FBW, WGR, SGR, FCR, and VSI ( $p>0.05)$. However, the CF was lower in the LBME, MBME, and HBME groups compared to the control group $(p<0.05)$. 
TABLE 2: Primer sequences for RT-qPCR detection of inflammatory cytokines genes.

\begin{tabular}{|c|c|c|c|c|c|}
\hline Gene & Primer & Sequence $\left(5^{\prime}-3^{\prime}\right)$ & Accession number & Product size & Amplification efficiency \\
\hline \multirow{2}{*}{$i l-1 \beta$} & $\mathrm{F}$ & TTACAGTAAGACCAGCCTGA & \multirow{2}{*}{ AJ245635 } & \multirow[t]{2}{*}{$91 \mathrm{bp}$} & \multirow[t]{2}{*}{$96 \%$} \\
\hline & $\mathrm{R}$ & AGGCTCGTCACTTAGTTTGT & & & \\
\hline \multirow{2}{*}{$i l-6$} & $\mathrm{~F}$ & GCAGCGCATCTTGAGTGTTTAC & \multirow{2}{*}{ AY102632 } & \multirow[t]{2}{*}{$73 \mathrm{bp}$} & \multirow[t]{2}{*}{$97 \%$} \\
\hline & $\mathrm{R}$ & CTGCTGCTCCATCACTGTCTTC & & & \\
\hline \multirow{2}{*}{$i l-8$} & $\mathrm{~F}$ & GTCTTAGAGGACTGGGTGTA & \multirow{2}{*}{ EU011243.1 } & \multirow[t]{2}{*}{$110 \mathrm{bp}$} & \multirow[t]{2}{*}{$98 \%$} \\
\hline & $\mathrm{R}$ & ACAGTGTGAGCTTGGAGGGA & & & \\
\hline \multirow{2}{*}{$i l-10$} & $\mathrm{~F}$ & CGCCAGCATAAAGAACTCGT & \multirow{2}{*}{ KX964678.1 } & \multirow[t]{2}{*}{$233 \mathrm{bp}$} & \multirow[t]{2}{*}{$96 \%$} \\
\hline & $\mathrm{R}$ & TGCCAAATACTGCTCGATGT & & & \\
\hline \multirow{2}{*}{$18 S$} & $\mathrm{~F}$ & CTGAGAAACGGCTACCACATC & \multirow[t]{2}{*}{ FJ710827.1 } & \multirow[t]{2}{*}{$107 \mathrm{bp}$} & \multirow[t]{2}{*}{$95 \%$} \\
\hline & $\mathrm{R}$ & GCCTCGAAAGAGACCTGTATTG & & & \\
\hline
\end{tabular}

TABLE 3: Growth performance of common carp (Cyprinus carpio L.) fed diets with different levels of BME for 8 weeks. Data are means of triplicate.

\begin{tabular}{lccccc}
\hline & Control & LBME & MBME & HBME & ANOVA $p$ value \\
\hline Initial body weight (IBM, g) & $13.95 \pm 0.26$ & $13.76 \pm 0.18$ & $14.14 \pm 0.06$ & $14.01 \pm 0.27$ & 0.377 \\
Final body weight (FBW, g) & $51.24 \pm 0.13$ & $52.86 \pm 2.23$ & $51.52 \pm 0.36$ & $52.00 \pm 0.17$ & 0.621 \\
Weight gain rate (WGR, \%) & $2.67 \pm 5.87$ & $2.75 \pm 2.51$ & $2.64 \pm 1.11$ & $2.71 \pm 1.65$ & 0.115 \\
Specific growth rate (SGR, \%/day) & $2.32 \pm 0.03$ & $2.40 \pm 0.06$ & $2.31 \pm 0.01$ & $2.34 \pm 0.01$ & 0.100 \\
Feed conversion ratio (FCR) & $1.05 \pm 0.02$ & $1.02 \pm 0.01$ & $1.06 \pm 0.00$ & $1.04 \pm 0.01$ & 0.119 \\
Condition factor (CF, g/cm ${ }^{3}$ ) & $3.05 \pm 0.05^{\mathrm{a}}$ & $2.60 \pm 0.04^{\mathrm{b}}$ & $2.59 \pm 0.02^{\mathrm{b}}$ & $2.63 \pm 0.07^{\mathrm{b}}$ & 0.000 \\
Viscera index (VSI, \%) & $9.01 \pm 1.32$ & $9.29 \pm 1.21$ & $9.56 \pm 1.72$ & $9.6 \pm 1.75$ & 0.853 \\
\hline
\end{tabular}

TABle 4: Serum antioxidant parameters of common carp (Cyprinus carpio L.) fed diets with different levels of BME for 8 weeks. Data are shown as mean \pm SEM $(n=9)$.

\begin{tabular}{lccccc}
\hline & Control & LBME & MBME & HBME & ANOVA $p$ value \\
\hline Superoxide dismutase (SOD, U/mL) & $0.74 \pm 0.47^{\mathrm{a}}$ & $2.07 \pm 0.57^{\mathrm{b}}$ & $2.41 \pm 0.16^{\mathrm{b}}$ & $1.74 \pm 0.25^{\mathrm{b}}$ & 0.002 \\
Catalase (CAT, U/mL) & $20.07 \pm 0.99^{\mathrm{a}}$ & $16.97 \pm 0.75^{\mathrm{a}}$ & $41.47 \pm 0.99^{\mathrm{b}}$ & $19.62 \pm 0.36^{\mathrm{a}}$ & 0.000 \\
Maleic dialdehyde (MDA, U/mL) & $17.69 \pm 4.49^{\mathrm{a}}$ & $14.85 \pm 3.35^{\mathrm{a}}$ & $24.81 \pm 8.78^{\mathrm{a}}$ & $6.33 \pm 2.77^{\mathrm{b}}$ & 0.000 \\
Total antioxidant capacity (T-AOC, U/mL) & $1.77 \pm 0.09^{\mathrm{a}}$ & $1.76 \pm 0.18^{\mathrm{a}}$ & $1.76 \pm 0.14^{\mathrm{a}}$ & $2.22 \pm 0.21^{\mathrm{b}}$ & 0.000 \\
\hline
\end{tabular}

3.2. Serum Oxidative and Antioxidant Parameters. The serum antioxidant parameters of fish in different groups are shown in Table 4. Compared with the control group, all the experimental groups (LBME, MBME, and HBME) with dietary BME supplementation significantly improved serum SOD activity $(p<0.05)$. Serum CAT activity in the MBME group was significantly higher compared with that in the control group $(p<0.05)$. Dietary supplementation with $1 \%$ BME (HBME group) significantly reduced MDA content and elevated T-AOC level in the serum of common carp $(p<0.05)$.

3.3. Expression of Inflammatory Cytokines. Dietary supplementation with BME at all proportions (LBME, MBME, and HBME diets) significantly inhibited $i l-1 \beta$ mRNA expression in the head kidney, spleen, and intestine of common carp $(p<0.05)$ (Figure 1(a)). The il-6 mRNA levels in the head kidney of fish fed with HBME diet were increased, and it was also elevated in the spleen of LBME, MBME and HBME groups and increased in the intestine of LBME group $(p<0.05)$ (Figure 1(b)). On the contrary, il-8 mRNA expression in the head kidney and spleen was downregulated significantly by dietary supplementation of BME at different levels $(p<0.05)$, and it was also inhibited in the intestine of LBME group $(p<0.05)$ (Figure $1(c))$. As for il-10, its mRNA level in the head kidney of fish fed with LBME and HBME diets was significantly higher than that of control group, and its level in the spleen of LBME and MBME groups as well as in the intestine of HBME group was also elevated remarkably $(p<0.05)$ (Figure $1(\mathrm{~d})$ ).

3.4. Intestinal Microbiota. As for intestinal microbiota analysis, a total of 2608 OTUs were obtained after the merge with a $97 \%$ species similarity. There were 1841, 1637, 1537, and 1532 OTUs in the control, LBME, MBME, and HBME groups, respectively. These OTUs were classified into 36 
$i l-1 \beta$

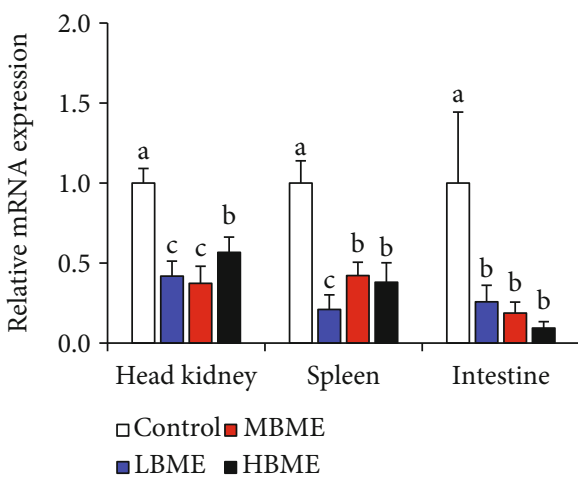

(a) $i l-8$

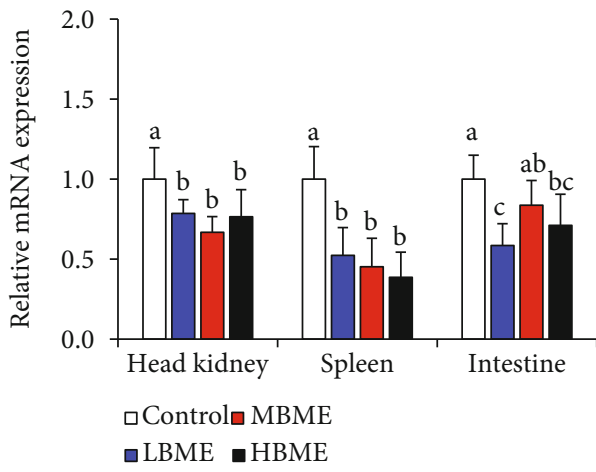

(c)

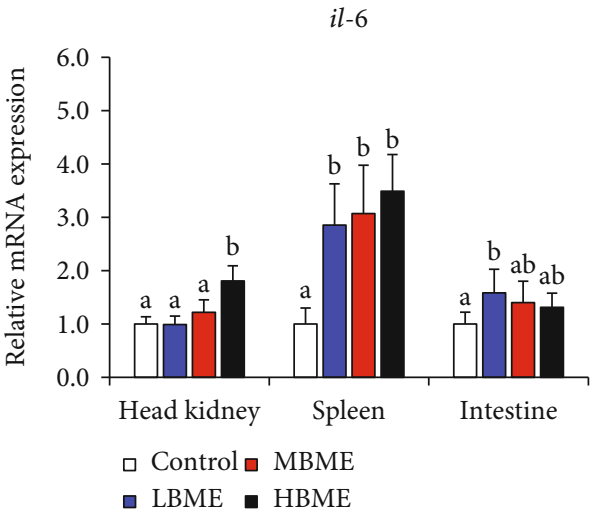

(b) il-10

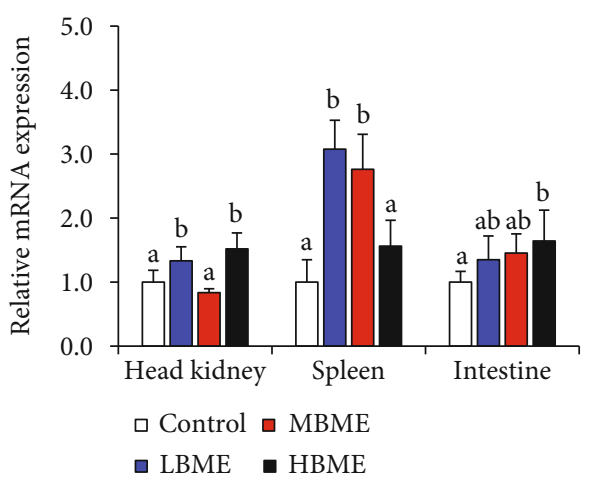

(d)

FIGURE 1: Effects of dietary bitter melon extract (BME) on gene expression of inflammatory cytokines in common carp (Cyprinus carpio L.). Fish were fed with either basal diets containing different proportions of BME $(0.25 \%, 0.5 \%$, and $1 \%$ for LBME, MBME, and HBME, respectively) or basal diet as control for 8 weeks. Gene expressions of $i l-1 \beta$ (a), il-6 (b), il-8 (c), and il-10 (d) were detected by RT-qPCR. Data are shown as mean \pm SEM $(n=9)$. Different letters in each column indicate significant differences $(p<0.05)$.

phylums, 79 classes, 216 orders, 396 families, and 871 genus. The OTUs from each sample were statistically analyzed based on the OTU table, and the Venn diagram was constructed. In this regard, 918 OTUs were shared by all gut samples of common carp fed with different diets, and the numbers of OTUs unique to control, LBME, MBME, and HBME groups were $316,230,134$, and 184, respectively (Figure 2(a)). Nonetheless, the principal coordinate analysis (PCoA) showed no differences in bacterial communities between the dietary BME groups and control group (Figure 2(b)), and there were no significant differences in Shannon index and Simpson index between the experimental groups and control group $(p>0.05)$ (Figure 2(c); Figure 2(d)). These results showed that intestinal microbial diversity was not affected by dietary BME.

To further understand the changes of microbial communities in the intestine of common carp, we analyzed its composition and relative abundance at phylum, family, and genus levels (Figure 3). At phylum level, the intestinal microbiota in each group was mainly composed of Proteobacteria, Actinobacteria, Chicroflexi, and Firmicutea, and the abundance of Firmicutes in the dietary BME treatment was relatively lower compared with that in control group $(p<0.05)$ (Figure 3(a)), and the ratio of Firmicutes to Bacter- oidetes was significantly decreased by dietary BME supplementation (Figure 3(b)). At family level, the relative abundance of streptococcaceae was decreased in the LBME, MBME, and HBME groups $(p<0.05)$ (Figure $3(c)$ ). At genus level, compared to the control group, dietary BME significantly reduced the relative abundances of Streptococcus $(p<0.05)$ and Exiguobacterium $(p<0.01)$ (Figure 3(d)).

\section{Discussion}

As a common vegetable, bitter melon and its extract (BME) have been widely concerned and used in traditional medicine due to its various biological activities [16]. However, its roles in aquatic animals have not been explored. In this study, we first evaluated the effect of dietary BME on growth performance of common carp. The result showed that growth parameters were unaffected by BME supplementation in the diet of common carp, including FBW, WGR, SGR, FCR, and VSI $(p>0.05)$. This suggested that BME had no growth-promoting effect on common carp. Consistent with our results, it was found that dietary BME supplementation had no significant effect on the body weight of rat fed with normal diet [24]. Although many studies have found that plant extracts effectively enhanced growth in 


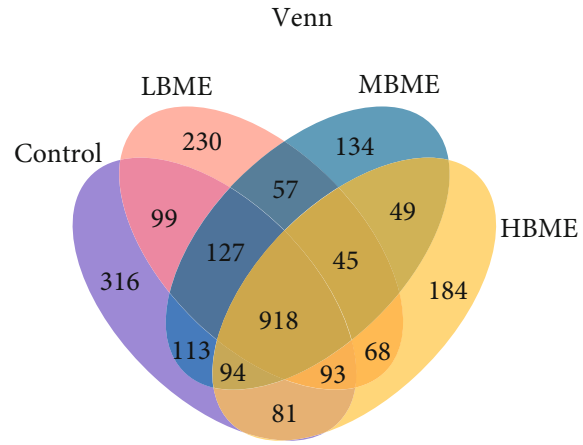

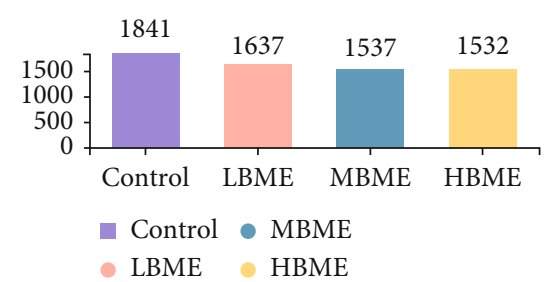

(a)

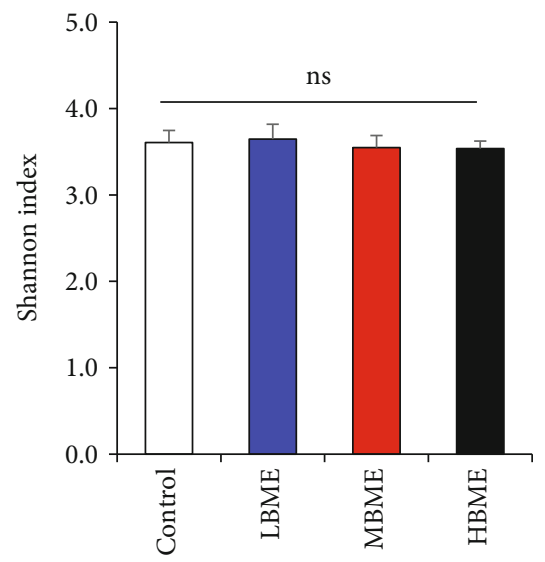

(c)

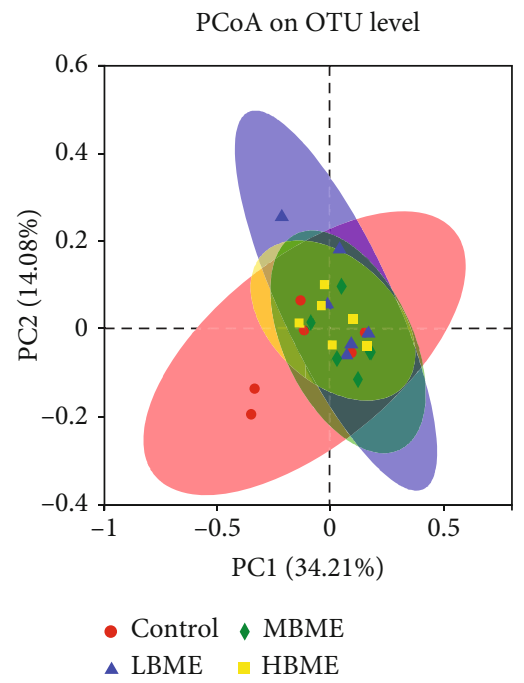

(b)

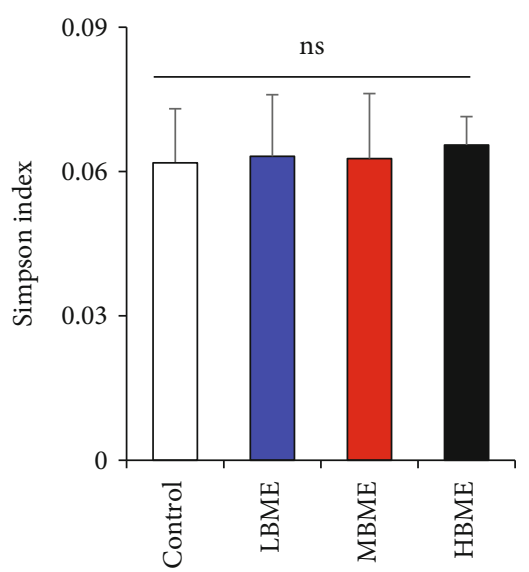

(d)

FIGURE 2: Effects of dietary bitter melon extract (BME) on the intestinal microbial diversity of common carp (Cyprinus carpio L.). (a) Venn diagram showing the distribution of OTUs in different groups. (b) Principal coordinate analysis (PCoA) of intestinal microbial communities. (c) and (d) Shannon index and Simpson index of the diversity of intestinal microbiota. "ns" represents that there was no significant difference among groups $(p>0.05)$.

aquatic animals, but it was also reported that some plant extracts had no growth-promoting effect. For example, honeysuckle (Lonicera japonica) extract and bee pollen extract did not significantly influence the growth performance of grass carp (Ctenopharyngodon idella) [25] and rainbow trout (Oncorhynchus mykiss) [26], respectively. This suggested that not all plant extracts could be acted as growth promoters, and their different effects on growth might be related to the active components. In addition, lower CF was caused by dietary BME in this study. This could be explained as the roles of BME in energy homeostasis. Studies in mice have shown that BME ameliorated obesity induced by high-fat diets [27].

Reactive oxygen species (ROS), which are the byproducts of normal physiological processes, react with a variety of biomolecules, and excessive ROS promotes lipid peroxidation, causes molecular damage, oxidative distress, and development of various diseases [28]. Antioxidant sys- tem protects cells from oxidative damage. SOD and CAT are important antioxidant enzymes involved in the resistance to harmful effects of free radicals, and the former contributes to the disputation of superoxide radicals to hydrogen peroxide and molecular oxygen, while the later catalyzes the reduction of hydrogen peroxides into water [29]. In the present study, dietary BME significantly increased serum SOD activity. Similarly, study in rat showed that BME treatment increased SOD and CAT activities in the heart tissue of diabetic rats [17] and inhibited stressinduced lipid peroxidation by increasing CAT activity [30]. However, serum CAT activity was only higher in the MBME group compared to that in the control group. This suggested that CAT activity in the serum of common carp was not influenced by low or high dietary BME levels. MDA is the product of lipid peroxidation, and its levels parallel the severity of free radicals attack, while T-AOC directly represents the antioxidant capacity of fish [31]. The present study 


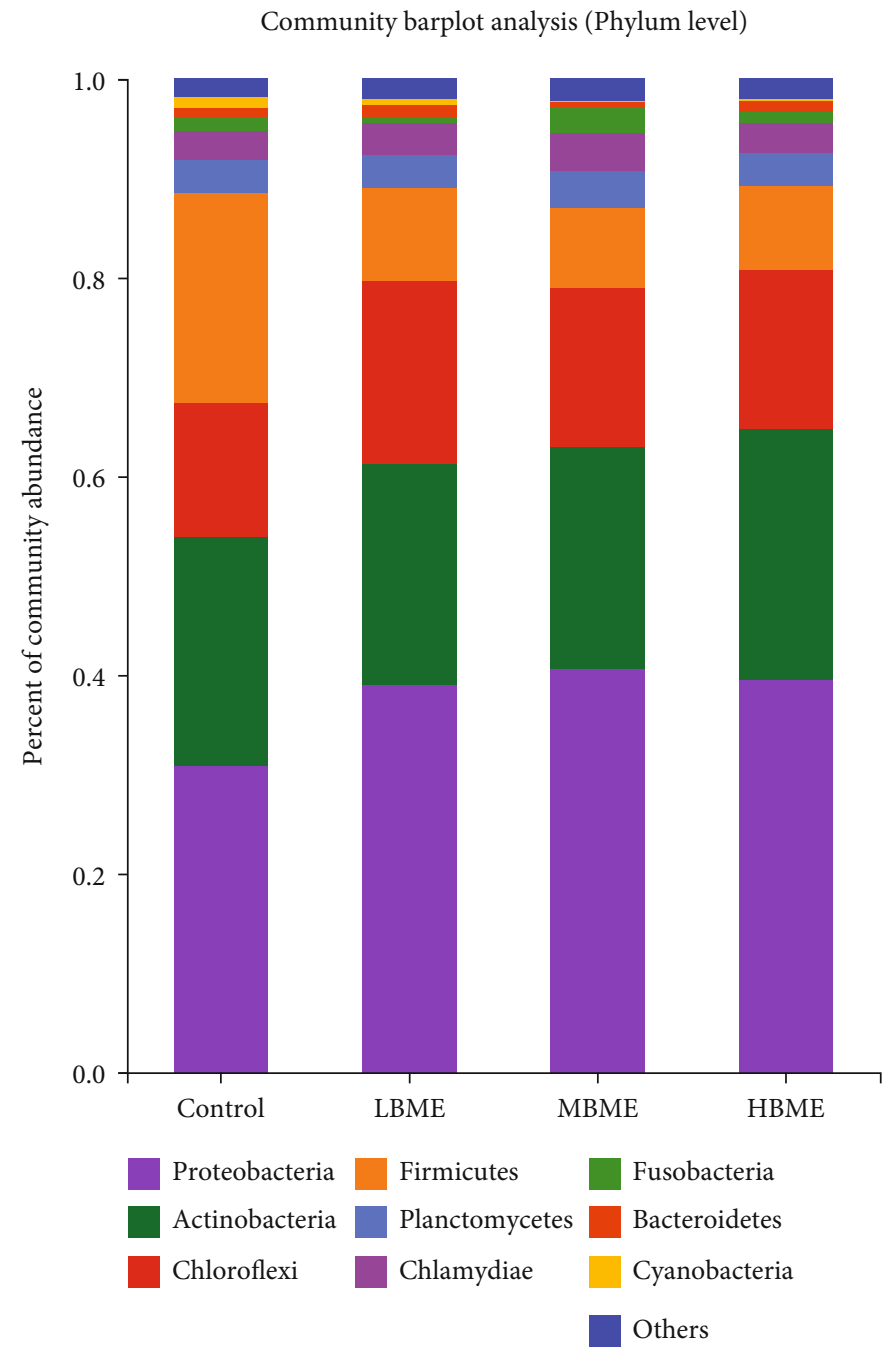

(a)

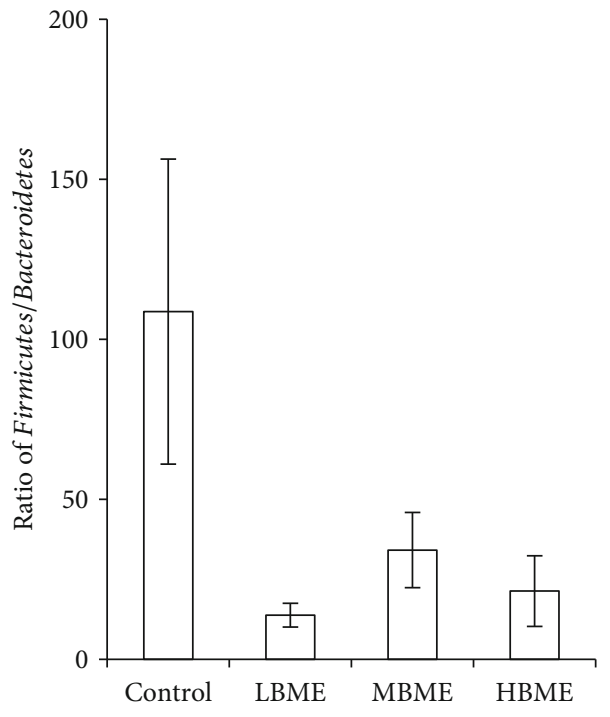

(b)

Figure 3: Continued. 


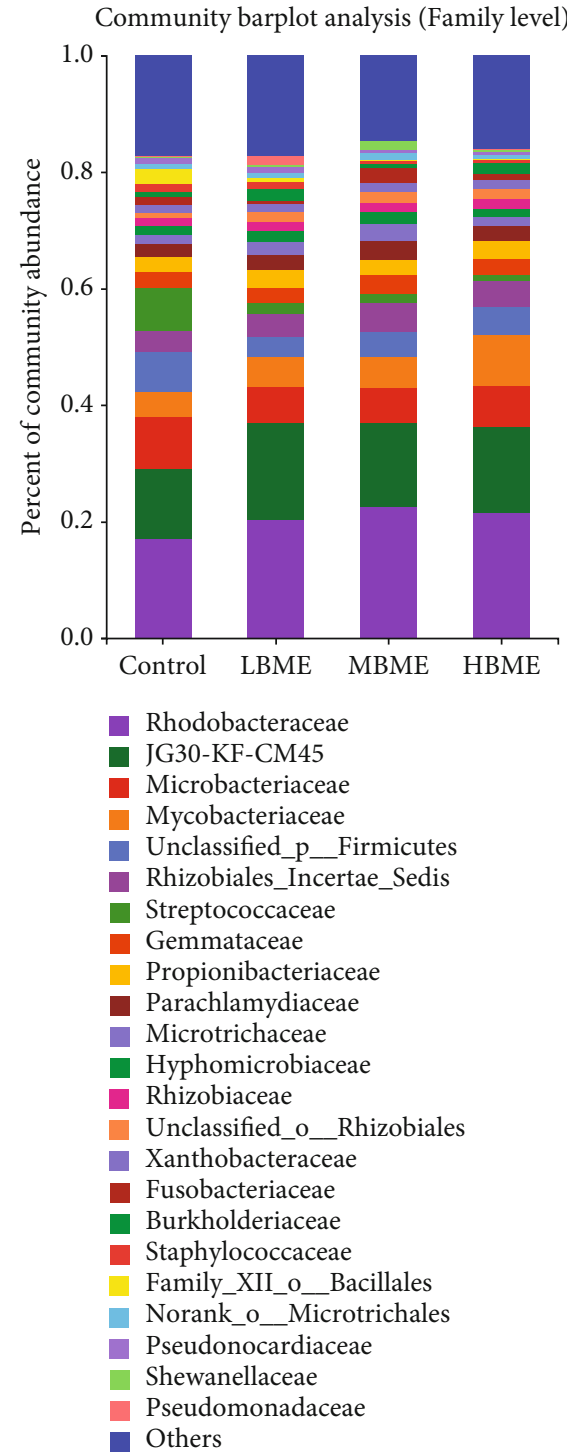

(c)

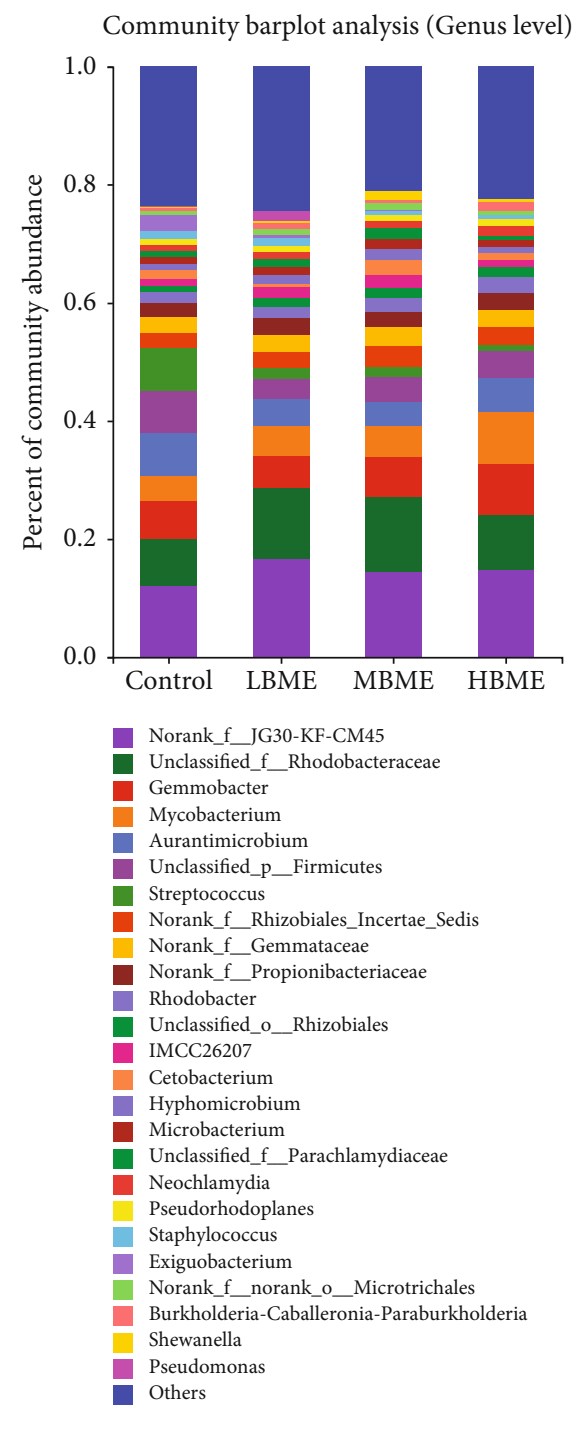

(d)

FIGURE 3: Composition and relative abundance of intestinal microbial communities of common carp (Cyprinus carpio L.) fed with different diets. (a), (c), and (d) The composition and relative abundance of bacterial communities at phylum, family, and genus level, and the relative abundance less than $0.01 \%$ was classified as others. (b) Ratio of Firmicutes to Bacteroidetes, different letters in each column indicate significant differences $(p<0.05)$.

showed that serum MDA content was significantly decreased in the HBME group, while serum T-AOC level was significantly elevated. These results suggested that dietary supplementation with BME improved the antioxidant capacity of common carp, and this point has been well demonstrated in some studies of rodents [32].

Immune status is closely related to inflammation, which is initiated and regulated by inflammatory cytokines [33]. IL- $1 \beta$ serves as a proinflammatory cytokine that activates innate immunity and promotes acute-phase response [34]. IL-6 is a soluble cytokine with pleiotropic activities, and it contributes to the host defense by inducing synthesis of acute phase proteins [35]. IL-8 is produced by various types of cells in response to inflammatory stimuli, and it plays an important role in the acute inflammation [36]. All these cytokines are considered to be proinflammatory factors in some fish species [37]. On the other hand, IL-10 exerts as anti-inflammatory cytokine in fish by inhibiting the production of reactive radicals and expression of proinflammatory cytokines [38]. It is reported that dietary supplementation with plant extracts can alter the transcriptional level of proinflammatory and anti-inflammatory cytokine genes in different organs of fish [39]. Bitter melon has been shown to have anti-inflammatory effects in mammals [40]. In this study, we measured the mRNA levels of inflammatory cytokine genes (il-1 $\beta, i l-6, i l-8$, and il-10) in response to BME supplementation in the diet of common carp.

The present study showed that dietary BME suppressed il-1 $\beta$ mRNA expression in the head kidney, spleen, and intestine of common carp and reduced il- 8 mRNA level in 
the head kidney and spleen. Similarly, a previous study found that BME reduced il-1 $\beta$ and il-6 mRNA expressions in the liver of mice [16], and bitter melon leaf extract suppressed il-1 $1 \beta$ and il-6 mRNA expressions in Propionibacterium acnes stimulated human monocytic THP- 1 cells in vitro [41]. These suggested that BME played a role in antiinflammation by inhibiting proinflammatory cytokine expression. As for il-6, however, the opposite result was found in this study. The il-6 mRNA expression in the spleen of common carp was upregulated by dietary BME at all proportions. Its mRNA level was also increased in the head kidney and intestine in the HBME and LBME groups, respectively. This could be explained as that the function of teleosts IL-6 differs from that of mammals. For example, il-6 mRNA level was downregulated by oral administration of LPS, which was opposite to what happened in mammals [42]. The il-10 mRNA abundance in the head kidney, spleen, and intestine of common carp was decreased by dietary BME at different proportions, and this result was similar to that previously reported. For example, adding wild bitter melon (Momordica charantia L. var. abbreviate Seringe) powder to the diet or treatment with bitter melon fruit juice significantly increased IL-10 secretion levels in rats [43, 44]. Likewise, oral administration of bitter melon powder also significantly improved serum IL-10 level of rats fed with high-fat diet [21]. This suggested that BME improved antiinflammatory ability of fish by promoting the expression of anti-inflammatory cytokine IL10.

Intestinal microbiota is well known as an important factor in regulating animal health, and it plays a pivotal role in the metabolism, inflammatory, and immune response of host [45]. Studies have shown that some plant extracts can significantly affect microbial communities in the intestine of aquatic animals. In this study, we found that there were no remarkable differences in Shannon index and Simpson index of the gut microbiota between the dietary BME groups and control group. PCoA result showed that the microbial communities in all groups were not significantly separated from each other. These results suggest that BME failed to affect the overall structure of the intestinal microbiota of common carp. This is in good agreement with previous findings in the literatures. For instance, bacterial taxa diversity in the intestine of rat with bitter melon powder (BMP) treatment was similar to that in the control group, but the gut microbiota in high-fat diet-induced obese rats were significantly modified, and the alpha diversity was increased by BMP intervention [21,46]. Dietary BME supplementation in the high-fat diets might have an effect on gut microbial diversity in fish, but it needs to be further validated.

At the level of phylum classification, the dominant communities in the intestine of common carp in all the groups were Proteobacteria, Actinobacteria, Chicroflexi, Firmicutea, Planctomycetes, Chlamydiate, Fusobacteria, and Bacteroidetes. This is consistent with what has been reported in previous studies [47]. In particular, dietary BME decreased the relative abundance of Firmicutes. This could be explained as a possible antibacterial activity of BME in common carp. This is because intestinal Firmicutes abundance was also decreased by dietary antibiotic mixture in the diet of grass carp [48]. Our result is also consistent with previous study, in which it was found that dietary Gelsemium elegans alkaloids reduced Firmicutes abundance in the gut of blunt snout bream (Megalobrama amblycephala) [49]. The ratio of Firmicutes to Bacteroidetes is closely related to obesity and intestinal health, and its value was significantly decreased in diabetic rats by treatment of bitter melon formulation [46]. In this study, similar results were obtained after the dietary BME treatment. The interaction between the relative abundance of Firmicutes to Bacteroides and energy metabolism as well as inflammatory response in fish needs to be further demonstrated. Streptococcaceae are known opportunistic pathogens, and its typical genus is Streptococcus [50]. Lower abundance of Streptococcaceae and Streptococcus was observed in the dietary BME groups. Similarly, resveratrol, a polyphenolic phytoalexin derived from plants, decreased the proportion of harmful microbial taxa (Streptococcaceae), whereas increased the abundance of beneficial microbial taxa (Acetobacteraceae and Methylobacteriaceae) in the intestine of tilapia (Oreochromis niloticus) [51]. Additionally, it was reported that dietary supplementation with Exiguobacterium acetylicum had a significant upregulation of $i$ - $1 \beta$ expression in the head kidney and spleen of goldfish (Carassius auratus) [52]. In line with this result, we found that dietary BME significantly reduced the abundance of Exiguobacterium at the genus level, while the il-1 $\beta$ mRNA expression level in the head kidney, spleen, and intestine was significantly elevated. Therefore, we speculated that the anti-inflammatory effect of BME could be closely related to its regulating effect on gut microbiota; however, the interaction between them remains to be elucidated.

\section{Conclusion}

In conclusion, dietary supplementation of BME had no effect on growth performance of common carp, but it elevated serum antioxidant capacity and inhibited the expression of proinflammatory cytokines (il-1 $\beta$ and $i l-8$ ), while the expression of anti-inflammatory cytokine (il-6 and il10) was promoted. In addition, the diversity of intestinal microbiota was not affected by dietary BME, but at phylum level, Firmicutes abundance and the ratio of Firmicutes to Bacteroidetes were reduced by dietary BME, and the relative abundance of streptococcaceae at family level and Streptococcus and Exiguobacterium at genus level was also decreased. Determination of the association between the antioxidant and anti-inflammatory effects of BME and its regulation on intestinal microbiota merits further study.

\section{Data Availability}

All data generated or used during the study appear in the submitted article.

\section{Conflicts of Interest}

The authors declare that they have no conflicts of interest. 


\section{Acknowledgments}

This study was supported by National Natural Science Foundation of China (31702358 and 31872581), Zhongyuan thousand talents plan-leading talents of Zhongyuan Science and Technology of Henan Province (204200510025), Science and Technology Breakthrough Major Project in Henan Province (212102110385), Innovation Scientists and Technicians Troop Construction Projects of Henan Normal University (2020TD02), Special Fund for Henan Agriculture Research System (S2014-10-G03).

\section{References}

[1] C. Chi, S. S. Giri, J. W. Jun et al., "Immunomodulatory effects of a bioactive compound isolated from Dryopteris crassirhizoma on the grass carp Ctenopharyngodon idella," Journal of Immunology Research, vol. 2016, Article ID 3068913, 10 pages, 2016.

[2] F. C. Cabello, "Heavy use of prophylactic antibiotics in aquaculture: a growing problem for human and animal health and for the environment," Environmental Microbiology, vol. 8, no. 7, pp. 1137-1144, 2006.

[3] M. Raissy, H. Ghafarifarsani, S. H. Hoseinifar, E. R. El-Haroun, S. Shahbazi Naserabad, and H. Van Doan, "The effect of dietary combined herbs extracts (oak acorn, coriander, and common mallow) on growth, digestive enzymes, antioxidant and immune response, and resistance against Aeromonas hydrophila infection in common carp, Cyprinus carpio," Aquaculture, vol. 546, article 737287, 2022.

[4] F. Zhu, "A review on the application of herbal medicines in the disease control of aquatic animals," Aquaculture, vol. 526, article 735422, 2020.

[5] T. Citarasu, "Herbal biomedicines: a new opportunity for aquaculture industry," Aquaculture International, vol. 18, no. 3, pp. 403-414, 2010.

[6] A. R. Hodar, R. Vasava, D. Mahavadiya, N. Joshi, V. Nandaniya, and H. Solanki, "Herbs and herbal medicines: a prominent source for sustainable aquaculture," Journal of Experimental Zoology India, vol. 24, pp. 719-732, 2021.

[7] M. Reverter, N. Bontemps, D. Lecchini, B. Banaigs, and P. Sasal, "Use of plant extracts in fish aquaculture as an alternative to chemotherapy: current status and future perspectives," Aquaculture, vol. 433, pp. 50-61, 2014.

[8] M. Yousefi, M. Naderi Farsani, H. Ghafarifarsani, S. H. Hoseinifar, and H. Van Doan, "The effects of dietary supplementation of mistletoe (Viscum album) extract on the growth performance, antioxidant, and innate, immune responses of rainbow trout (Oncorhynchus mykiss)," Aquaculture, vol. 536, article 736385, 2021.

[9] S. M. Hoseini, A. Taheri Mirghaed, Y. Iri, S. H. Hoseinifar, H. van Doan, and M. Reverter, "Effects of dietary Russian olive, Elaeagnus angustifolia, leaf extract on growth, hematological, immunological, and antioxidant parameters in common carp, Cyprinus carpio," Aquaculture, vol. 536, article 736461, 2021.

[10] G. Mohammadi, G. Rashidian, S. H. Hoseinifar, S. S. Naserabad, and H. V. Doan, "Ginger (Zingiber officinale) extract affects growth performance, body composition, haematology, serum and mucosal immune parameters in common carp (Cyprinus carpio)," Fish \& Shellfish Immunology, vol. 99, pp. 267-273, 2020.
[11] X. H. Tan, Z. Z. Sun, M. Zhou et al., "Effects of dietary hawthorn extracts supplementation on lipid metabolism, skin coloration and gut health of golden pompano (Trachinotus ovatus)," Aquaculture, vol. 519, article 734921, 2020.

[12] J. L. Wang, X. L. Meng, R. H. Lu et al., "Effects of Rehmannia glutinosa on growth performance, immunological parameters and disease resistance to Aeromonas hydrophila in common carp (Cyprinus carpio L.)," Aquaculture, vol. 435, pp. 293300, 2015.

[13] X. L. Meng, W. P. Hu, S. K. Wu et al., "Chinese yam peel enhances the immunity of the common carp (Cyprinus carpio L.) by improving the gut defence barrier and modulating the intestinal microflora," Fish \& Shellfish Immunology, vol. 95, pp. 528-537, 2019.

[14] K. Abascal and E. Yarnell, "Using bitter melon to treat diabetes," Alternative and Complementary Therapies, vol. 11, no. 4, pp. 179-184, 2005.

[15] S. Jia, M. Shen, F. Zhang, and J. Xie, "Recent advances in Momordica charantia: functional components and biological activities," International Journal of Molecular Sciences, vol. 18, no. 12, p. 2555, 2017.

[16] P. R. Dandawate, D. Subramaniam, S. B. Padhye, and S. Anant, "Bitter melon: a panacea for inflammation and cancer," Chinese Journal of Natural Medicines, vol. 14, no. 2, pp. 81-100, 2016.

[17] U. N. Tripathi and D. Chandra, "The plant extracts of Momordica charantia and Trigonella foenum graecum have antioxidant and anti-hyperglycemic properties for cardiac tissue during diabetes mellitus," Oxidative Medicine and Cellular Longevity, vol. 2, no. 5, 296 pages, 2009.

[18] B. Bao, Y. G. Chen, L. Zhang et al., "Momordica charantia (bitter melon) reduces obesity-associated macrophage and mast cell infiltration as well as inflammatory cytokine expression in adipose tissues," PLoS One, vol. 8, no. 12, article e84075, 2013.

[19] A. R. Wang, C. Ran, E. Ring $\varnothing$, and Z. G. Zhou, "Progress in fish gastrointestinal microbiota research," Reviews in Aquaculture, vol. 10, no. 3, pp. 626-640, 2018.

[20] F. Chen, Q. Wen, J. Jiang et al., "Could the gut microbiota reconcile the oral bioavailability conundrum of traditional herbs?," Journal of Ethnopharmacology, vol. 179, pp. 253264, 2016.

[21] J. Bai, Y. Zhu, and Y. Dong, "Response of gut microbiota and inflammatory status to bitter melon (Momordica charantia L.) in high fat diet induced obese rats," Journal of Ethnopharmacology, vol. 194, pp. 717-726, 2016.

[22] G. Qiao, P. Chen, Q. R. Sun et al., "Poly- $\beta$-hydroxybutyrate (PHB) in bioflocs alters intestinal microbial community structure, immune-related gene expression and early cyprinid herpesvirus 2 replication in gibel carp (Carassius auratus gibelio)," Fish \& Shellfish Immunology, vol. 97, pp. 72-82, 2020.

[23] C. B. Qin, W. L. Zhao, G. K. Yang et al., "Hepatic glucagon receptors expression in response to glucose, insulin, glucagon and immune challenge in grass carp (Ctenophcuyngodon idellus)," Aquaculture Reports, vol. 18, article 100470, 2020.

[24] G. V. K. Senanayake, N. Fukuda, S. Nshizono et al., "Mechanisms underlying decreased hepatic triacylglycerol and cholesterol by dietary bitter melon extract in the rat," Lipids, vol. 47, no. 5, pp. 495-503, 2012.

[25] X. L. Meng, Z. X. Zhu, R. H. Lu et al., "Regulation of growth performance and lipid metabolism in juvenile grass carp 
(Ctenopharyngodon idella) with honeysuckle (Lonicera japonica) extract," Fish Physiology and Biochemistry, vol. 45, no. 5, pp. 1563-1573, 2019.

[26] E. G. T. Sanchez, C. A. Fuenmayor, S. M. V. Mejia, C. DiazMoreno, and H. S. Mahecha, "Effect of bee pollen extract as a source of natural carotenoids on the growth performance and pigmentation of rainbow trout (Oncorhynchus mykiss)," Aquaculture, vol. 514, article 734490, 2020.

[27] M. A. Alam, R. Uddin, N. Subhan, M. M. Rahman, P. Jain, and H. M. Reza, "Beneficial role of bitter melon supplementation in obesity and related complications in metabolic syndrome," Journal of Lipids, vol. 2015, Article ID 496169, 18 pages, 2015.

[28] C. A. Ferreira, D. Ni, Z. T. Rosenkrans, and W. Cai, "Scavenging of reactive oxygen and nitrogen species with nanomaterials," Nano Research, vol. 11, no. 10, pp. 4955-4984, 2018.

[29] M. S. Talkad, A. Javed, M. Jaiswal, and B. M. Shruthi, "Protective effect of Salacia Oblanga and quercetin on cyclophosphamide-induced chromosome aberrations in rat bone marrow cells," International Journal of Engineering Inventions, vol. 2, no. 5, pp. 36-43, 2013.

[30] P. Chaturvedi, "Bitter melon protects against lipid peroxidation caused by immobilization stress in albino rats," International Journal for Vitamin and Nutrition Research, vol. 79, no. 1, pp. 48-56, 2009.

[31] B. Wang, Y. Liu, L. Feng et al., "Effects of dietary arginine supplementation on growth performance, flesh quality, muscle antioxidant capacity and antioxidant-related signalling molecule expression in young grass carp (Ctenopharyngodon idella)," Food Chemistry, vol. 167, pp. 91-99, 2015.

[32] S. Wang, Z. Li, G. Yang, C. T. Ho, and S. Li, "Momordica charantia: a popular health-promoting vegetable with multifunctionality," Food \& Function, vol. 8, no. 5, pp. 1749-1762, 2017.

[33] L. Chen, L. Feng, W. D. Jiang et al., "Intestinal immune function, antioxidant status and tight junction proteins mRNA expression in young grass carp (Ctenopharyngodon idella) fed riboflavin deficient diet," Fish \& Shellfish Immunology, vol. 47, no. 1, pp. 470-484, 2015.

[34] D. T. Cooke, E. G. Hoyt, and R. C. Robbins, "Overexpression of human Bcl-2 in syngeneic rat donor lungs preserves posttransplant function and reduces intragraft caspase activity and interleukin- $1 \beta$ production," Transplantation, vol. 79, no. 7, pp. 762-767, 2005.

[35] T. Tanaka, M. Narazaki, and T. Kishimoto, "IL-6 in inflammation, immunity, and disease," Cold Spring Harbor Perspectives in Biology, vol. 6, no. 10, article a016295, 2014.

[36] A. Harada, N. Sekido, T. Akahoshi, T. Wada, N. Mukaida, and K. Matsushima, "Essential involvement of interleukin-8 (IL-8) in acute inflammation," Journal of Leukocyte Biology, vol. 56, no. 5, pp. 559-564, 1994.

[37] T. G. Choudhury and D. Kamilya, "Paraprobiotics: an aquaculture perspective," Reviews in Aquaculture, vol. 11, no. 4, pp. 1258-1270, 2019.

[38] M. C. Piazzon, H. F. J. Savelkoul, D. Pietretti, G. F. Wiegertjes, and M. Forlenza, "Carp Il10 has anti-inflammatory activities on phagocytes, promotes proliferation of memory $\mathrm{T}$ cells, and regulates B cell differentiation and antibody secretion," The Journal of Immunology, vol. 194, no. 1, pp. 187-199, 2015.

[39] Y. L. Jiang, S. S. Feng, S. H. Zhang et al., “Transcriptome signatures in common carp spleen in response to Aeromonas hydrophila infection," Fish \& Shellfish Immunology, vol. 57, pp. 4148, 2016.
[40] W. S. Yang, E. Yang, M. J. Kim et al., "Momordica charantia inhibits inflammatory responses in murine macrophages via suppression of TAK1," The American Journal of Chinese Medicine, vol. 46, no. 2, pp. 435-452, 2018.

[41] W. C. Huang, T. H. Tsai, C. J. Huang et al., "Inhibitory effects of wild bitter melon leaf extract on Propionibacterium acnesinduced skin inflammation in mice and cytokine production in vitro," Food \& Function, vol. 6, no. 8, pp. 2550-2560, 2015.

[42] T. Kadowaki, Y. Yasui, O. Nishimiya et al., "Orally administered LPS enhances head kidney macrophage activation with down-regulation of IL-6 in common carp (Cyprinus carpio)," Fish \& Shellfish Immunology, vol. 34, no. 6, pp. 1569-1575, 2013.

[43] C. Y. Chao, P. J. Sung, W. H. Wang, and Y. H. Kuo, "Antiinflammatory effect of Momordica charantia in sepsis mice," Molecules, vol. 19, no. 8, pp. 12777-12788, 2014.

[44] R. Fachinan, A. Yessoufou, M. P. Nekoua, and K. Moutairou, "Effectiveness of antihyperglycemic effect of Momordica charantia: implication of T-cell cytokines," Evidence-based Complementary and Alternative Medicine, vol. 2017, Article ID 3707046, 8 pages, 2017.

[45] J. B. Xiong, L. Nie, and J. Chen, "Current understanding on the roles of gut microbiota in fish disease and immunity," Zoological Research, vol. 40, no. 2, pp. 70-76, 2019.

[46] Y. Zhu, J. Bai, Y. Zhang, X. Xiao, and Y. Dong, "Effects of bitter melon (Momordica charantia L.) on the gut microbiota in high fat diet and low dose streptozocin-induced rats," International Journal of Food Sciences and Nutrition, vol. 67, no. 6, pp. 686695, 2016.

[47] D. Z. Xie, Q. Y. Dai, C. Xu, and Y. Y. Li, "Dietary tributyrin modifies intestinal function by altering morphology, gene expression and microbiota profile in common carp (Cyprinus carpio) fed all-plant diets," Aquaculture Nutrition, vol. 27, no. 2, pp. 439-453, 2021.

[48] J. J. Tian, Y. Q. Jin, E. M. Yu et al., "Farnesoid X receptor is an effective target for modulating lipid accumulation in grass carp, Ctenopharyngodon idella," Aquaculture, vol. 534, article 736248, 2021.

[49] Q. Ye, Y. Y. Feng, Z. L. Wang et al., "Effects of dietary Gelsemium elegans alkaloids on intestinal morphology, antioxidant status, immune responses and microbiota of Megalobrama amblycephala," Fish \& Shellfish Immunology, vol. 94, pp. 464-478, 2019.

[50] H. H. Xu, Y. Y. Wei, H. Q. Ma et al., "Alterations of gut microbiome in the patients with severe fever with thrombocytopenia syndrome," Frontiers in Microbiology, vol. 9, p. 2315, 2018.

[51] Y. Zheng, W. Wu, G. D. Hu et al., "Gut microbiota analysis of juvenile genetically improved farmed tilapia (Oreochromis niloticus) by dietary supplementation of different resveratrol concentrations," Fish \& Shellfish Immunology, vol. 77, pp. 200-207, 2018.

[52] S. Jinendiran, A. A. Nathan, D. Ramesh, B. Vaseeharan, and N. Sivakumar, "Modulation of innate immunity, expression of cytokine genes and disease resistance against Aeromonas hydrophila infection in goldfish (Carassius auratus) by dietary supplementation with Exiguobacterium acetylicum S01," Fish \& Shellfish Immunology, vol. 84, pp. 458-469, 2019. 